\title{
Isolamento e identificação de amebas de vida livre potencialmente patogênicas em amostras de ambientes de hospital público da Cidade de Porto Alegre, RS
}

\author{
Isolation and identification of potentially pathogenic free-living amoebae in samples \\ from environments in a public hospital in the City of Porto Alegre, Rio Grande do Sul
}

\author{
Ana Maris Carlesso ${ }^{1,2}$, Amauri Braga Simonetti ${ }^{3}$, Geórgia Lazzari Artuso ${ }^{3}$ \\ e Marilise Brittes Rott $^{3}$
}

\begin{abstract}
RESUMO
Um estudo sobre a presença de amebas de vida livre em um hospital público foi desenvolvido na Cidade de Porto Alegre, RS. Poeira e biofilmes de 15 ambientes hospitalares, inchindo CTI, UTI pediátrica, cozinha, emergência, centro cirúrgico ambulatorial e centro cirúrgico, reservatórios de água, torneira e 6 bebedouros coletivos foram coletados mensalmente, de julbo de 2004 a março de 2005, usando-se suabes estéreis, preparados para a pesquisa. As AVLforam isoladas em cultivo, utilizando-se meio de ágarnão nutriente adicionado de Escherichia coli, mortaspelo calor:A identificação dos protozoários foi feita pela observação morfológica de cistos e trofozoitos, segundo critérios morfológicos de Page (1988). Das 135 amostras coletadas dos 15 ambientes estudados, 47 (35\%) foram positivas para AVL. Destas, 34\% apresentaram características morfológicas próprias do gênero Acanthamoeba.
\end{abstract}

Palavras-chaves: Amebas de vida livre. Poeira. Biofilmes. Ambiente hospitalar. Acanthamoeba.

\begin{abstract}
A study on the presence of free-living amoebae in a public hospital was developed in the city of Porto Alegre, State of Rio Grande do Sul. Dust and biofilms were collected using sterile swabs that had been prepared for this study, from 15 hospital environments, including the intensive care center, pediatric intensive care unit, kitchen, emergency room, outpatient surgical center, clinical surgical center, water storage tanks, taps and six drinking fountains for general use, every month from July 2004 to March 2005. The FLAs were isolated by culturing, using nonnutrient agar medium with the addition of heat-killed Escherichia coli. The protozoa were identified by morphological observation of cysts and trophozoites, in accordance with Page's morphological criteria (1988). Among the 135 samples collected from the 15 environments, 47 (35\%) were positive for FLAs. Of these, thirty-four percent presented morphological characteristics particular to the genus Acanthamoeba.
\end{abstract}

Key-words: Free-living amoebae. Dust. Biofilm. Hospital environment. Acanthamoeba.

As amebas de vida livre (AVL), que esporadicamente parasitam o homem, são protozoários com ampla dispersão ambiental, isoladas em lagos, rios, piscinas, solos, esgotos e cursos de água que recebem efluentes industriais, estando presente em todos os continentes e climas ${ }^{14913162124252734}$. Também, são encontradas em água para consumo humano, descargas termais, equipamentos lava-olhos, encanamentos de equipo odontológico e unidades de hemodiálise 35252630 .

As três principais AVL potencialmente patogênicas para 0 homem e animais são: Naegleria fowleri, Acanthamoeba spp e Balamuthia mandrillaris, as quais têm sido relacionadas com meningoencefalites, ulcerações da pele e infecções da córnea.
Naegleria fowleri a única espécie que causa em humanos a meningoencefalite amebiana primária (MAP), uma infecção nãooportunística, mas de curso rápido e fatal. Geralmente acomete crianças e jovens saudáveis com histórias de terem nadado dias antes em lagos ou piscinas ${ }^{14101829313336}$. Acanthamoeba spp e Balamuthia mandrillaris podem causar encefalite amebiana granulomatosa (EAG), uma infecção considerada oportunista, pois quase todos os casos relatados até o momento se referem a indivíduos imunologicamente debilitados, especialmente àqueles que sofreram algum tipo de terapia ou situação imunossupressora, como alcoolismo, gravidez, quimioterapia ou uso de antibióticos de amplo espectro. Mais recentemente, vêem sendo descritos casos

1. Curso de Pós-Graduação em Microbiologia Agrícola e do Ambiente, Universidade Federal do Rio Grande do Sul, Porto Alegre, RS. 2. Hospital de Clínicas de Porto Alegre, Porto Alegre, RS. 3. Departamento de Microbiologia, Universidade Federal do Rio Grande do Sul, Porto Alegre, RS.

Financiado pelo CNPq.

Endereço para correspondência: Dra. Ana Maris Carlesso. Av. João Pessoa 721/607, 90040-000 Porto Alegre, RS.

Tel: $55513286-6361$

e-mail: anacarlesso@yahoo.com.br; marilise.rott@ufrgs.br

Recebido para publicação em 3/7/2006

Aceito em 2/5/2007 
de infecção por Acanthamoeba spp. e Balamuthia mandrillaris em pacientes com AIDS 261519222931 . A ceratite por Acanthamoeba tem se mostrado como um crescente problema no campo da oftalmologia, ocorrendo principalmente em usuários de lentes de contato, embora dados recentes indiquem que possa estar associada com outras condições predisponentes. 0 diagnóstico desta ameba na superfície ocular é freqüientemente difícil de ser realizado, pois sua apresentação pode mimetizar ceratite herpética ou bacteriana. 0 atraso no diagnóstico pode ser extremamente prejudicial ao paciente, conduzindo ao agravamento do quadro podendo culminar até mesmo em enucleação ${ }^{11} 14151737$. Assim, o encontro de AVL potencialmente patogênicas em amostras ambientais às quais o ser humano se expõe com frequiência, é de grande interesse para determinação de riscos, decorrente dessa exposição. AVL em ambientes hospitalares se mostram como possíveis fontes de infecções aos seres humanos ${ }^{162526}$. Tem sido demonstrada a resistência dos seus cistos a desinfetantes, como o cloro, dióxido de cloro, ozônio, deciquam 222 entre outros, dificultando assim a desinfecção de ambientes contaminados. Além disso, as AVL resistem a condições extremas de temperatura $\mathrm{e} \mathrm{pH}^{7132630}$. 0 gênero Acanthamoeba, também tem sido implicado como veículo de várias bactérias patogênicas, como por exemplo, a Legionella spp., entre outros endossimbiontes ${ }^{20} 291$. No Brasil, os poucos trabalhos realizados até o momento são insuficientes para demonstrar a real importância das amebas de vida livre como possíveis agentes patogênicos, principalmente em ambientes hospitalares. Baseado no exposto, realizou-se estudo com o objetivo de verificar a ocorrência de AVLs no Hospital de Clínicas de Porto Alegre, avaliando-se a poeira de diversos ambientes hospitalares, além dos biofilmes de reservatórios e bebedouros coletivos.

\section{MATERIAL E MÉTODOS}

Foram coletadas 135 amostras de poeira e biofilmes entre os meses de julho de 2004 e março de 2005 em 15 ambientes (Centro de Terapia Intensiva, Centro Cirúrgico, UTI Pediátrica, Cozinha, Emergência, Centro Cirúrgico Ambulatorial, 02 Reservatórios, seis Bebedouros coletivos e uma torneira da cozinha) do Hospital de Clínicas de Porto Alegre, RS (HCPA). As amostras dos ambientes selecionados foram coletadas com suabes esterilizados e secos, que foram passados de forma aleatória sobre locais das áreas escolhidas, como o piso, janelas, móveis, biofilme de bebedouros coletivos, da torneira e dos reservatórios de água. As amostras foram processadas segundo Silva \& Rosa ${ }^{31}$. Para o isolamento das amebas, foi utilizado o meio de ágar não-nutriente ${ }^{2328} 1,5 \%$ recoberto com uma suspensão de Escherichia coli, mortas pelo calor. A seguir as placas foram seladas com Parafilm ${ }^{\circledR} \mathrm{e}$ incubadas a $30^{\circ} \mathrm{C}$ por até 10 dias. A verificação do crescimento amebiano foi realizada diariamente colocando-se a placa invertida sobre a platina de um microscópio óptico usando a objetiva de 10X. A identificação das amebas baseou-se no tipo de movimento e nos critérios morfológicos dos cistos e trofozoítos segundo Page $(1988)^{23}$. A identificação de Naegleria fowleri foi realizada através da técnica de exflagelação e confirmada em triplicata. Após o crescimento de trofozoítos, a superfície das placas de ágar nãonutriente foi coberta com $10 \mathrm{~mL}$ de água destilada estéril e raspada cuidadosamente com alça de platina. 0 líquido foi colocado em garrafas estéreis de cultivo $\left(25 \mathrm{~cm}^{3}\right)$ que foram incubadas a $37^{\circ} \mathrm{C}$. As garrafas foram examinadas em microscópio invertido para verificar a emissão de flagelos a cada 30 min durante 4 horas. As amostras negativas foram observadas por até 24 horas e, então, descartadas $^{831}$.

\section{RESULTADOS}

Das 135 amostras de poeira e biofilmes, coletadas dos 15 ambientes hospitalares selecionados, 47 (35\%) foram positivas para amebas de vida livre (AVL). Foram isoladas amebas de vida livre em todos os ambientes analisados, exceto no centro de tratamento intensivo (CTI). Dois tipos de material foram coletados: poeira e biofilmes. Das 47 (35\%) amostras positivas para AVL, $11(23,4 \%)$ foram de poeira provenientes de cinco ambientes: cozinha, emergência, centro cirúrgico ambulatorial, centro cirúrgico, e UTI pediátrica e $36(76,6 \%)$ de biofilmes provenientes de seis bebedouros, dois reservatórios de água e uma torneira da cozinha. Os bebedouros 8 e 14 foram os que apresentaram a maior contaminação por AVL, com 14 amostras positivas dentre as 18 coletadas. A Tabela 1 resume os resultados obtidos nas nove coletas referentes à presença de AVL nos ambientes hospitalares investigados.

Tabela 1 - Número e percentual de amostras positivas para amebas de vida livre em diferentes ambientes hospitalares, no período de julbo/04 a março/05.

\begin{tabular}{|c|c|c|c|}
\hline \multirow[b]{2}{*}{ Ambiente } & \multirow[b]{2}{*}{$\begin{array}{c}\text { Amostras } \\
\text { positivas } \\
\mathrm{n}^{0}\end{array}$} & \multicolumn{2}{|c|}{ Percentual (\%) de amostras positivas } \\
\hline & & $\begin{array}{l}\text { em relação às coletas } \\
\text { do ambiente }(\mathrm{n}=9)\end{array}$ & $\begin{array}{l}\text { em relação às coletas } \\
\text { do hospital }(\mathrm{n}=135)\end{array}$ \\
\hline Cozinha & 6 & 66,7 & 4,4 \\
\hline Centro cirúrgico & 1 & 11,1 & 0,7 \\
\hline UTI pediátrica & 1 & 11,1 & 0,7 \\
\hline CCA & 1 & 11,1 & 0,7 \\
\hline Emergência & 2 & 22,2 & 1,5 \\
\hline CTI & 0 & 0 & 0 \\
\hline Bebedouro 08 & 7 & 77,8 & 5,2 \\
\hline Bebedouro 13 & 2 & 22,2 & 1,5 \\
\hline Bebedouro 14 & 7 & 77,8 & 5,2 \\
\hline Bebedouro 15 & 5 & 55,6 & 3,7 \\
\hline Bebedouro 19 & 6 & 66,7 & 4,4 \\
\hline Beb emerg* & 2 & 22,2 & 1,5 \\
\hline Reserv azul** & 4 & 44,4 & 3 \\
\hline Reserv conc** & 1 & 11,1 & 0,7 \\
\hline Torneira/cozinha & 2 & 22,2 & 1,5 \\
\hline Total & 47 & 53 & 35 \\
\hline
\end{tabular}


Das 47 amostras positivas para AVL, 16 (34\%) são sugestivas de pertencer ao gênero Acanthamoeba. Os isolados apresentaram trofozoítos com locomoção lenta, citoplasma com zona periférica hialina, a partir de onde se notaram projeções hialinas e delgadas, os filopódios ou acantopódios e a presença de vacúolos pulsáteis ou contráteis no citoplasma. Os cistos apresentaram parede com membrana dupla, sendo o endocisto triangular, estrelado ou poligonal e o ectocisto enrugado. Considerando-se o total de amostras coletadas, 11,9\% dos isolados apresentaram características do gênero Acanthamoeba. Os locais de onde foram isoladas as amebas de vida livre sugestivas de pertencerem ao gênero Acanthamoeba, bem como seus percentuais em relação ao total de amostras coletadas $(n=135)$ e ao total de amostras positivas $(n=47)$ estão representados na Tabela 2 .

A cozinha foi o segundo ambiente que apresentou o maior índice de contaminação por AVL. Neste local, quando da realização das coletas, também observou-se a presença de umidade e um número grande de pessoas circulando. Também, observaram que Acanthamoeba spp foi isolada em 4 coletas realizadas no reservatório de azulejo, localizado no subsolo e que a água deste reservatório é distribuída para todo o hospital. Dos 47 isolados de AVL, provenientes dos ambientes hospitalares, nenhum apresentou exflagelação.

\begin{tabular}{|c|c|c|c|}
\hline \multirow{2}{*}{$\begin{array}{l}\text { Ambientes } \\
\text { Hospitalares }\end{array}$} & \multirow{2}{*}{$\begin{array}{l}\text { Número de isolados sugestivos de } \\
\text { Acanthamoeba }\end{array}$} & \multicolumn{2}{|c|}{ Percentual de amostras (\%) } \\
\hline & & coletadas & positivas \\
\hline B14 & 1 & 0,7 & 2,1 \\
\hline $\mathrm{CC}$ & 1 & 0,7 & 2,1 \\
\hline CCA & 1 & 0,7 & 2,1 \\
\hline $\mathrm{CZ}$ & 6 & 4,4 & 12,8 \\
\hline EM & 2 & 1,5 & 4,2 \\
\hline RA & 4 & 3,0 & 8,5 \\
\hline UTI & 1 & 0,7 & 2,1 \\
\hline Total & 16 & 11,8 & 34 \\
\hline
\end{tabular}

B: bebedouro; CC: centro cirúrgico; CCA: centro cirúrgico ambulatorial; CZ: cozinha; EM: emergência; RA: reservatório azulejo; UTI: unidade de terapia intensiva

\section{DISCUSSÃO}

Os relatos sobre o isolamento de amebas de vida livre potencialmente patogênicas a partir de poeira são escassos. Kingston \& Warhurst ${ }^{16}$ isolaram essas amebas da poeira e do ar de um quarto ocupado por crianças acometidas de infecção respiratória. Foram utilizadas amostras coletadas através da aspiração do ar ambiente. Os autores isolaram 12 cepas de amebas do gênero Acanthamoeba e 3 de Naegleria. Giazzi ${ }^{12}$ estudando a prevalência de amebas de vida livre potencialmente patogênicas em vários habitats na cidade de Araraquara, São Paulo, coletou 23 amostras de poeira, obtendo um percentual de $86,9 \%$ para espécies de Acanthamoeba, e de 39,1\% para espécies de Naegleria. Em um estudo, realizado por Rohr e cols ${ }^{26}$, em 6 hospitais na Alemanha, foram encontradas 29 (52\%) amostras positivas para amebas de vida livre em 56 amostras de água coletadas de sistemas de água quente e 23 (47\%) de 49 amostras coletadas com suabes das áreas úmidas de sanitários. Nesse estudo, os autores identificaram grande variedade de espécies de AVL presentes nas áreas úmidas, com 41 organismos identificados pertencentes a 6 diferentes gêneros. Acanthamoeba e Naegleria foram os gêneros mais isolados, ambos com percentual de $22 \%$. Silva \& Rosa ${ }^{32}$ isolaram AVL potencialmente patogênicas em $60(45,5 \%)$ amostras de poeira de um total de 132, coletadas em dois hospitais da Cidade de Presidente Prudente, SP. Obtiveram de 72 amostras de poeira coletadas no Hospital Universitário, 30 (41,6\%) positivas para AVL potencialmente patogênicas. No segundo, Hospital Estadual, foram coletadas 60 amostras de poeira, sendo observadas AVL potencialmente patogênicas em 30 (50\%) das amostras. Em outro estudo, Barbeau \& Buhler ${ }^{3}$ analisando amostras de biofilmes de 35 equipos dentários e de 18 torneiras, relataram o isolamento de AVL nos 53 ambientes coletados. Em relação à concentração de AVL isoladas dos biofilmes, os autores chegaram à conclusão de que os equipos dentários tinham uma concentração de AVL três vezes mais alta do que nas amostras de torneira. No presente estudo, foram obtidos 36 isolados de AVL de 6 bebedouros, uma torneira e de 2 reservatórios de água avaliados, sendo, portanto as amostras de biofilmes as que apresentaram maior percentual de positividade quando comparadas às de poeira. Embora, os autores do trabalho anteriormente mencionado não tenham coletado amostras de poeira, a positividade em biofilmes para AVL é quase sempre $100 \%$ segundo trabalhos citados na literatura ${ }^{52637}$ e a explicação para esse fato, é que AVL se aderem aos biofilmes para se alimentar das bactérias que os colonizam. Os autores do estudo citado acima, ainda relatam que os equipos dentários tiveram significante positividade, devido esse material ter um diâmetro menor para passagem de líquidos fazendo com que o contato de bactérias com os equipos seja grande, com isso criando biofilmes em toda extensão da tubulação. Esta explicação está em concordância com nossos resultados para as amostras de biofilme, pois nossas coletas nos bebedouros foram realizadas na saída de água de menor diâmetro. No estudo de Silva \& Rosa ${ }^{31}$, os autores avaliaram a presença de AVL somente na poeira de diversos ambientes hospitalares e verificaram maior positividade na cozinha e no berçário, provavelmente devido à maior umidade desses locais e também devido ao maior número de pessoas circulando no ambiente.

0 menor índice de positividade no referido trabalho ocorreu no centro cirúrgico e UTI, ambientes onde existe um maior cuidado com a limpeza e há um número restrito de profissionais circulando. Os resultados do presente trabalho concordam em relação à positividade de alguns ambientes igualmente selecionados, especialmente em relação à menor positividade, que ocorreu a exemplo do trabalho de Silva \& Rosa ${ }^{31}$, em ambientes como o centro cirúrgico, UTI pediátrica e centro de tratamento intensivo. Os resultados, provavelmente se devem ao fato de que nesses locais, por abrigarem pacientes de risco, além da maior preocupação com limpeza e desinfecção, o acesso de pessoas é normalmente restrito a poucos profissionais, que fazem uso de equipamentos de proteção individual, como luvas, máscaras, gorros, protetores de pés e roupas apropriadas e esterilizadas. 
Esses cuidados poderiam contribuir para a diminuição da contaminação desses ambientes, já que se sabe que espécies de Acanthamoeba têm sido isoladas como flora microbiota normal das vias aéreas superiores de indivíduos saudáveis ${ }^{18}$.

Diversos autores concordam que existe certa dificuldade em isolar Naegleria fowleri do ambiente ${ }^{24}$. Pesquisas realizadas nos mais diversos ambientes, em geral mostram percentuais bem menores de isolados de Naegleria em relação a outras AVL ${ }^{35}$. Uma explicação para os baixos índices de recuperação de Naegleria fowleri do ambiente seria sua fragilidade. Cursons e $\mathrm{cols}^{7}$, estudando a resistência das AVL frente ao efeito de alguns desinfetantes como Deciquam 222, cloro, dióxido de cloro e ozônio, verificaram que todos demonstraram ação desinfetante contra as AVL, porém, eram necessárias concentrações mais altas do que as usadas contra as bactérias. No presente estudo quando foram realizadas as coletas, houve a informação de que era feita limpeza com hipoclorito a $1 \%$ no reservatório de concreto a cada seis meses e nos bebedouros era usado hipoclorito a $5 \%$, cada vez que era percebido gosto metálico na água. 0 uso freqüente desse desinfetante em alguns locais poderia explicar a ausência de isolados de Naegleria no ambiente hospitalar. As primeiras amostras positivas para o gênero Acanthamoeba no reservatório de água apareceram quando já se fazia necessária nova limpeza e aplicação de hipoclorito a $1 \%$. 0 estudo realizado por Silva \& Rosa ${ }^{32}$ em poeira de ambientes hospitalares na Cidade de Presidente Prudente, SP, entre os meses de agosto e novembro de 2002, mostrou 45,5\% de Acanthamoeba spp e 3,8\% de Naegleria spp. No referido trabalho, foi usada a técnica de exflagelação para identificar 0 gênero Naegleria. A explicação dos autores para seus achados baseou-se no fato de que os cistos de Naegleria são poucos resistentes à dessecação e a desinfetantes, fazendo com que apareçam menos em ambientes mais secos ou sujeitos a desinfecções periódicas ${ }^{293}$.

Os resultados encontrados no presente estudo são semelhantes àqueles encontrados pela maioria dos autores que estudaram AVL nos mais variados ambientes, sendo o gênero Acanthamoeba 0 mais isolado mundialmente entre as AVL, principalmente devido à resistência de seus cistos às variações ambientais $3^{36152936}$. É preocupante a ocorrência dessas amebas, já que muitos pacientes internados em hospitais têm sua imunidade deprimida, seja naturalmente devido a doenças ou a pouca idade, ou artificialmente pelo uso de drogas imunossupressoras. Outros são submetidos a cirurgias ou podem, ainda, apresentar problemas de queimadura em áreas extensas da pele, o que pode facilitar a implantação de germes oportunistas, entre os quais, espécies de Acanthamoeba.

\section{REFERÊNCIAS}

1. Abraham SN, Lawande RV. Incidence of free-living amoebae in the nasal passages of local population in Zaria, Nigeria. Journal of Tropical Medicine and Hygiene 85:217-222, 1982.

2. Anzil AP, Rao C, Wrzolek MA, Visvesvara GS, Sher JH, Kozlowski PB. Amebic meningoencephalitis in a patient with AIDS caused by a newly recogized opportunistic pathogen: Leptomyxid ameba. Archives of Pathology \& Laboratory Medicine 115: 21-25, 1991.
3. Barbeau J, Buhler T. Biofilmes augment the number of free-living amoebae in dental unit waterlines. Research in Microbiology 152:753-760, 2001.

4. Carter RF. Primary amoebic meningoencephalitis: Clinical pathological and epidemiological features of six fatal cases. Journal of Pathology and Bacteriology 96: 1-25, 1968.

5. Casemore DP. Free-living amoebae in home dialysis unit. Journal Lancet 2:1078, 1977.

6. Chappell CL, Wright JA, Coletta M, Newsome AL. Standardized method of measuring Acanthamoeba antibodies in sera from healthy human subjects. Clinical and Diagnostic Laboratory Immunology 8:724-730, 2001.

7. Cursons RTM, Brown TJ, Keys EA. Effect of disinfectants on pathogenic free-living ameobae: in axenic condiction. Applied and Environmental Microbiology 40: 62-66, 1980 .

8. De Carli GA. Parasitologia Clínica: Seleção e Métodos e Técnicas de Laboratório para Diagnóstico das Parasitoses Humanas. Atheneu, São Paulo, 2001.

9. De Jonckheere JF. Ecology of Acanthamoeba. Reviews of Infectious Diseases 13: 385-387, 1991.

10. Ferrante A. Free-living amoebae: pathogenicity and immunity. Parasite Immunology 13: 31-47, 1991.

11. Ficker L, Seal D, Warhurst D, Wright P. Acanthamoeba keratitis: resistance to medical therapy. Eye 4:835-838, 1990.

12. Giazzi JF. Contribuição para o estudo do isolamento, cultivo e manutenção das amebas de vida livre. Tese (Livre Docência) - Faculdade de Ciências Farmacêuticas, Universidade Estadual Paulista, Araraquara, SP, 1996.

13. Griffin JL. Temperature tolerance of pathogenic and non pathogenic free-living amoebas. Science Journal 178: 869-870, 1972.

14. Illinworth CD, Cook SD. Acanthamoeba keratitis. Survey of Ophthalmology 42:493-508, 1998.

15. Khan NA. Pathogenesis of Acanthamoeba infections. Microbial Pathogenesis 34:277-285, 2003.

16. Kingston D, Warhurst DC. Isolation of amoebae from the air. Journal Medical Microbiology 2:27-36, 1969.

17. Ma P, Willaert E, Juechter KB, Stevens AR. A case of keratitis due to Acanthamoeba in New York, New York, and features of 10 cases. The Journal of Infectious Diseases 143: 662-667, 1981.

18. Martinez AJ. Free-living amebas: infection of the central nervous system. The Mount Sinai Journal Medicine 60:271-278, 1993.

19. Martinez AJ, Janitschke K. Acanthamoeba, an opportunistic microorganism: a review. Infection 13: 251-256, 1985.

20. Molmeret M, Horn M, Wagner M, Santic M, Kwaik YA. Amoebae as training grounds for intracellular bacterial pathogens. Applied and Environmental Microbiology 71: 20-28, 2005.

21. Morales JL, Rivas AO, Foronda P, Martínez E, Valladares B. Isolation and identification of pathogenic Acanthamoeba strains in Tenerife, Canary Islands, Spain from water sources. Parasitology Research 95:273-277, 2005.

22. Murakawa GJ, Mccalmont T, Altman J, Telang GH, Hoffman MD, Kantor GR, Berger TG. Disseminated acanthamebiasis in patients with AIDS. Archives of Dermatology 131:1291-1296, 1995.

23. Page FC. A New Key to Freshwater and Soil Gymnamoebae. Freshwater Biological Association, Ambleside 1988.

24. Pernin P, Pélandakis M, Rouby Y, Faure A, Siclet F. Comparative Recoveries of Naegleria fowleri Amoebae from Seeded River Water by Filtration and Centrifugation. Applied and Environmental Microbiology 64: 955-959, 1998.

25. Rivera F, Ramírez E, Bonilla P, Calderón A, Gallegos E, Rodríguez S, Ortiz R, Zaldívar B, Ramírez P, Durán A. Pathogenic and free-living amoebae isolated from swimming pools and physiotherapy tubs in Mexico. Environmental Research 62:43-52, 1993 .

26. Rohr U, Weber S, Michel R, Selenka F, Wilhelm M. Comparison of free-living amoebae in hot water systems of hospitals with isolates from moist sanitary areas by identifying genera and determining temperature tolerance. Applied and Environmental Microbiology 64: 1822-1824, 1998.

27. Sawyer TK. Free-living pathogenic and nonpathogenic amoebae in Maryland soils. Applied and Environmental Microbiology 55: 1074-1077, 1989. 
28. Schuster FL. Cultivation of pathogenic and opportunistic free-living amebas. Clinical Microbiology Reviews 15: 342-354, 2002.

29. Schuster FL, Visvesvara GS. Free-living amoebae as opportunistic and nonopportunistic pathogens of humans and animals. Journal of Parasitology 34:1001$1027,2004$.

30. Sheehan KB, Fagg JA, Ferris MJ, Henson JM. PCR detection and analysis of the freeliving amoeba Naegleria in hot springs in Yellowstone and Grand Teton National Parks. Applied and Environmental Microbiology 69:5914-5918, 2003.

31. Silva MA. Isolamento e identificação de amebas de vida livre potencialmente patogênicas em amostras de poeira de hospitais da cidade de Presidente Prudente. Dissertação de Mestrado, Faculdade de Ciências Farmacêuticas, Universidade Estadual Paulista, Araraquara, SP, 2001

32. Silva MA, Rosa JA. Isolamento de amebas de vida livre potencialmente patogênica em poeira de hospitais. Revista de Saúde Pública 37: 242-246, 2003.
33. Singh BN, Das SR. Intra-nasal infection of mice with flagellate stage of Naegleria aerobia and its bearing on the epidemiology of human meningo-encephalitis. Currente Science 41: 625-628, 1972

34. Stevens AR, Tyndall RL, Coutant CC, Willaert E. Isolation of the etiological agent of primary amoebic meningoencephalitis from artificially heated waters. Applied and Environmental Microbiology 34: 701-705, 1977.

35. Tsvetkova N, Schild M, Panaiotov S, Kurdova-Mintcheva R, Gottstein B, Walonik J, Aspöck H, Lucas MS, Müller N. The identification of free-living environmental isolates of amoebae from Bulgaria. Parasitology Research 92: 405-413, 2004.

36. Visvesvara GS, Stehr-Green JK. Epidemiology of free-living ameba infections. Journal of Parasitology 37:25S-33S, 1990

37. Walochnik J, Haller-Shober EM, Kölli H, Picher O, Obwaller A, Aspöck H Discrimination between clinically relevant an nonrelevant Acanthamoeba strains isolated from contact lens-wearing keratitis patients in Austria. Journal of Clinical Microbiology 38: 3932-3936, 2000 